\title{
Evaluation of CRRT effects on pyemic secondary AKI by serum cartilage glycoprotein 39 and Annexin A1
}

\author{
YU WU ${ }^{1}$, LING WANG $^{1}$, LEI MENG $^{3}$, GUANG-KE CAO $^{1}$ and YANG ZHANG ${ }^{2}$ \\ ${ }^{1}$ Department of Nephrology, The First People's Hospital of Xuzhou, Xuzhou, Jiangsu 221000; \\ ${ }^{2}$ Department of Anesthesiology, Xuzhou Medical University, Xuzhou, Jiangsu 221004; \\ ${ }^{3}$ Department of Intensive Care Unit, The Affiliated Hospital of Xuzhou Medical University, \\ Xuzhou, Jiangsu 221000, P.R. China
}

Received January 25, 2016; Accepted August 24, 2016

DOI: 10.3892/etm.2016.3691

\begin{abstract}
The aim of the present study was to examine the effects of continuous renal replacement therapy (CRRT) on pyemic secondary acute kidney injury (AKI) by serum cartilage glycoprotein 39 (YKL-40) and Annexin A1. From October, 2013 to October, 2015, 45 pyemic secondary AKI cases and 40 pyemic non-secondary AKI cases were selected for the present study. There were also 35 cases of physical examination volunteers. The serum YKL-40 and Annexin A1 levels were compared.CRRT was applied to pyemic secondary AKI patients and based on the obtained results the patients were divided into the success and failure groups. YKL-40, Annexin A1, hs-CRP, creatinine and urea nitrogen levels after $1,6,12,24,48$ and 72 h of AKI were measured. The YKL-40 and Annexin A1 levels in the pyemic secondary AKI group were significantly higher than those in other two groups and differences were statistically significant $(\mathrm{P}<0.05)$. There was no statistically significant difference regarding time period for applying CRRT in the success and failure groups $(\mathrm{P}>0.05)$. The peak level of YKL-40 and Annexin A1 in the success group decreased more rapidly compared to the failure group and the difference was statistically significant $(\mathrm{P}<0.05)$. When the differences in creatinine and urea nitrogen levels at different time points were compared between the success and failure groups, no statistical significance was observed $(\mathrm{P}>0.05)$. However, the success group showed a significantly lower level compared to the failure group at $72 \mathrm{~h}$. Comparisons for other time periods showed no statistical significance $(\mathrm{P}>0.05)$. Thus, the serum cartilage glycoprotein 39 and Annexin A1 level were able to predict the clinical effects of CRRT on pyemic secondary AKI.
\end{abstract}

Correspondence to: Dr Yang Zhang, Department of Anesthesiology, Xuzhou Medical University, 209 Tongshan Road, Xuzhou, Jiangsu 221004, P.R. China

E-mail: zhang_yang_1212@163.com

Key words: cartilage glycoprotein 39, Annexin A1, pyemia, acute kidney injury, continuous renal replacement therapy

\section{Introduction}

The incidence of pyemic secondary acute kidney injury (AKI) is extremely high at $50-80 \%$ (1). It is mostly believed that pathogenesis is correlated with: i) specific tissue structure of kidney; ii) ischemia reperfusion; iii) inflammation caused by endotoxin; and iv) gene expression theory (2). Several RCT studies have confirmed that early continuous renal replacement therapy (CRRT) is able to improve clinical symptoms and reverse disease course $(3,4)$. Based on the RENAL study conducted in 2009 and CRRT study performed on 1,349 cases of comprehensive JSEPTIC and BEST databases in 2013, the use of high dose of CRRT did not produce better results compared to a low dose of CCRT $(5,6)$. It was also suggested that AKI patients receive CRRT as early as possible before any obvious complications appear. Indexes such as blood urea nitrogen, creatinine and urine volume serve only as references for CRRT, and uniform and ideal clinical criteria and reference index are lacking.

Pyemia and AKI were shown to be linked to inflammatory response and imbalance $(6,7)$. Ronco et al found that hs-CRP was an acute phase reactive protein and CRRT was capable of reducing the hs-CRP level, which was closely associated with prognosis (8). Nevertheless, no final conclusion has been drawn regarding wehther hs-CRP is able to guide CRRT. Cartilage glycoprotein 39 (YKL-40) and Annexin A1 are two newly identified inflammatory factors, with possible links to the incidence and development of pyemia and AKI (9). To the best of our knowledge, few studies have demonstrated whether these proteins can be used as sensitive and specific indexes for CRRT.

The aim of the present study was to examine the value of applying YKL-40 and Annexin A1 in guiding CRRT based on monocentric and small sample clinical controlled trials.

\section{Materials and methods}

Object data. From October, 2013 to October, 2015, 45 pyemic secondary AKI cases as well as 40 pyemic non-secondary AKI cases were selected. There were also 35 cases of physical examination volunteers. Pyemia was diagnosed in accordance with diagnostic criteria jointly prepared by the USA SCCM, 
ACCP, ATS, ESICM and SIS. AKI was based on diagnostic criteria recommended by KDIGO (10).

Inclusion criteria for the study were: i) patients were $\geq 18$ years but $<75$ years; ii) they were all diagnosed in accordance with CRRT indexes and features suggested by KDIGO; and iii) they all had complete clinical data.

Exclusion criteria for the study were: i) none of our cases had primary renal diseases such as nephrotic syndrome; history of renal transplantation, solitary kidney and congenital nephrarctia; ii) none of them had septic shock, multiple resistant bacteria, severe underlying diseases such as heart failure, liver failure, malignant tumor and coagulation disorders; iii) patients with $<12$ months to live were excluded; and iv) pregnant women, patients with autoimmune disease and non-compliant patients were also excluded. Signed written informed consent was obtained from all the participants prior to the study. The study was approved by the Ethics Committee of the First People's Hospital of Xuzhou.

For pyemic secondary AKI patients, there were 28 males and 17 females; aged between 29 and 68 years, with an average age of $45.7 \pm 15.6$ years. Of these there were 22 cases of drugresistant bacteremia, 8 cases of infection after injury, 5 cases of infection after burn, 4 cases of infection after severe acute pancreatitis and 6 cases of infection after autoimmune disease drugs. For pyemic non-secondary AKI patients, there were 25 males and 15 females; aged between 28 and 69 years, with an average age of $46.3 \pm 13.8$ years. Of these, there were 20 cases of drug-resistant bacteremia, 7 cases of infection after injury, 3 cases of infection after burn, 5 cases of infection after severe acute pancreatitis and 5 cases of infection after autoimmune disease drugs. The physical examination volunteers group comprised 23 males and 12 females; aged 24-67 years, with an average age of $43.8 \pm 14.4$ years. Differences of age and gender in the three groups were not statistically significant. Differences between types of infection in the pyemic secondary and nonsecondary AKI group were not statistically significant.

Study method. Treatment was started based on the 'CRRT application indexes and features suggested by KDIGO' (10). Reasonable sepsis bundle was also applied. The bundle included: i) initial fluid resuscitation; ii) antibiotic; iii) vasoactive agent; and iv) active correction on primary diseases. Additionally, glucocorticoid, blood transfusion and mechanical ventilation adjuvant therapy were also applied when necessary. CVVH was used in CRRT and blood flow volume was $180-200 \mathrm{ml} / \mathrm{min}$ and the flow was $35-40 \mathrm{ml} / \mathrm{kg} \cdot \mathrm{h}$. Substitution fluid was $0.9 \%$ sodium chloride $(3,000 \mathrm{ml})+$ water $(1,000 \mathrm{ml})+5 \%$ glucose $(250 \mathrm{ml})+10 \%$ calcium chloride $(10 \mathrm{ml})+50 \%$ magnesium sulfate $(1.8 \mathrm{ml})+5 \%$ sodium bicarbonate $(250 \mathrm{ml})+10 \%$ potassium chloride $(15 \mathrm{ml})$. Prior to initiation of the experiment, unfractionated heparin (25,000 units) was used to prime blood filter and blood filter line and low molecular heparin 6,000 units to maintain anticoagulation and monitor coagulation function. APTT equal to 1.5- to 2-fold over the normal value was considered appropriate and this was filtered for 8-12 h each time. The main instruments included in the study were: i) Baxter Aquarius CRRT machine and ii) Aqu-line disposable blood purification tube (both from Baxter, Deerfield, IL, USA); iii) 12 Fr double channel deep vein catheter (Arrow

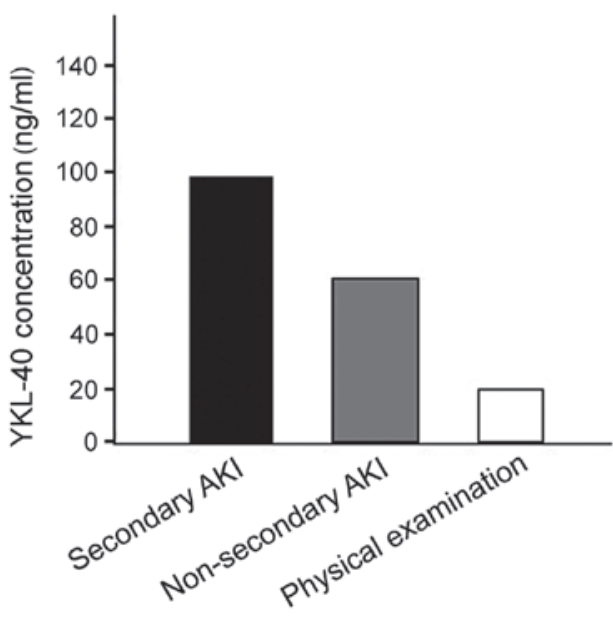

Figure 1. Determination of YKL-40 concentration using ELISA.

Medical, Kington, Herefordshire, UK); and iv) Baxter blood filtration apparatus HF 1200 (Baxter).

Observation index and testing method. Serum YKL-40 and Annexin A1 levels in the three groups were compared. CRRT was used for pyemic secondary AKI patients, who were divided into the success and failure groups based on results. YKL-40, Annexin A1, hs-CRP, creatinine and urea nitrogen levels were compared after 1, 6, 12, 24, 48 and $72 \mathrm{~h}$ of AKI.

Separation of mononuclear cells from peripheral serum: Anticoagulant blood $(5 \mathrm{ml})$ was centrifuged for $20 \mathrm{~min}$ at $1,500 \mathrm{x} \mathrm{g}$ and the supernatant was discarded. Hank solution $(2.5 \mathrm{ml})$ was added to the pellet based on a volume ratio of 1:1 provided by Sangon Biotech Co., Ltd. (Shanghai, China). Subsequently $5 \mathrm{ml}$ of LTS1077 lymphocytes separating solution (Sigma, Darmstadt, Germany) was added and centrifuged for $20 \mathrm{~min}$ at $1,500 \mathrm{x}$ g. Plasma was removed and Hank solution was added to the PBMC. The mix was subsequently centrifuged for $10 \mathrm{~min}$ at $1,000 \mathrm{x} \mathrm{g}$. RPMI-1640 medium (Sigma) was used to rinse the pellets to remove impurities such as blood platelet fully.

YKL-40 concentration was tested using ELISA. PBMC separating medium $(1 \mathrm{ml})$ was incubated for $30 \mathrm{~min}$ at $37^{\circ} \mathrm{C}$. Serum was centrifuged for $10 \mathrm{~min}$ at $2,500 \mathrm{x} \mathrm{g}$ and the supernatant was removed. The YKL-40 ELISA kit (no. 8020; Quidel Corp., San Diego, CA, USA) was used.

Western blot analysis was used to test the Annexin A1 expression level. Total protein in PBMC was extracted using the conventional protein extraction method. After SDS-PAGE, the proteins were transferred to a nitrocellulose membrane. The membrane was blocked for $2 \mathrm{~h}$ (5\% skim milk), and primary rabbit polyclonal Annexin A1 antibody (Abcam, Cambridge, MA, USA; catalog no. ab33061; dilution, 1:1,000) were added and incubated at $4^{\circ} \mathrm{C}$ overnight. The membrane was then rinsed and secondary horseradish peroxidase coupled with goat anti-rabbit antibody was added (Abcam; catalog no. ab6721; dilution, 1:5,000). After 1-h incubation at $25^{\circ} \mathrm{C}$, DAB development was used and the gel image processing system (Denver Company, CO, USA) was employed for quantitative analysis ( $\beta$-globin expression level as internal control). The protein expression level was shown as the density ratio between the targeted gene and $\beta$-globin. 


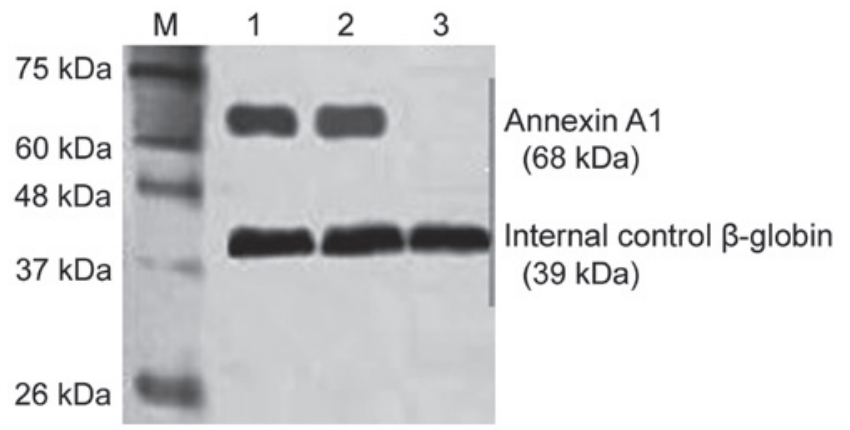

Figure 2. The level of A1 protein expression shown by western blot analysis. Lane 1, secondary AKI group; lane 2, non-secondary AKI group; and lane 3, healthy physical examination group. AKI, acute kidney injury.

Statistical method. SPSS 19.0 statistical software (Chicago, IL, USA) was used for data analysis. Measurement data were presented as mean value \pm standard deviation, analysis of variance (ANOVA) was applied for comparisons among groups. The independent sample t-test was used for comparisons between two groups. Comparison of different points within one group were carried out using repeated measurement data. Enumeration data were shown as case or (\%), and the $\chi^{2}$ test was used for comparisons between groups. $\mathrm{P}<0.05$ was considered statistically significant.

\section{Results}

Comparison of YKL-40 and Annexin Al level in the three groups. YKL-40 level in the pyemic secondary AKI group was obviously higher than those in the other two groups and differences were statistically significant $(\mathrm{P}<0.001)$. The YKL-40 level was $105.7 \pm 26.3 \mathrm{ng} / \mathrm{ml}$ in the pyemic secondary AKI group and $62.4 \pm 22.9$ and $18.9 \pm 6.6 \mathrm{ng} / \mathrm{ml}$ in the other two groups.

The Annexin A1 level was also significantly higher in the pyemic secondary AKI group compared with the other two groups $(0.853 \pm 0.076 \mathrm{ng} / \mathrm{ml}$ vs. $0.582 \pm 0.055 \mathrm{ng} / \mathrm{ml}$ and $0.043 \pm 0.022 \mathrm{ng} / \mathrm{ml})$. Differences were statistically significant $(\mathrm{P}<0.001)$ (Figs. 1 and 2).

Comparison of YKL-40 and Annexin Al levels between the success and failure secondary AKI group. There were 30 success cases together with 15 failure cases in the secondary AKI group. Of these cases, 5 individuals in the failure group succumbed, and there were 8 cases of acute renal failure and 2 cases of respiratory failure. Differences in the time period of using CRRT in the success and failure groups showed no statistical significance. YKL-40 and Annexin A1 expression levels in the success group were decreased more significantly and the difference was statistically significant $(\mathrm{P}<0.05)$ (Fig. 3).

Comparisons of the $h s-C R P$, creatinine and urea nitrogen level. Differences between the creatinine and urea nitrogen levels in the success and failure groups, at all time points, were not statistically significant $(\mathrm{P}>0.05)$. The levels of creatinine and urea nitrogen in the success group were significantly decreased as compared to the failure group at $72 \mathrm{~h}$, while comparisons at other time points showed no statistical significance (P>0.05) (Fig. 4).
A

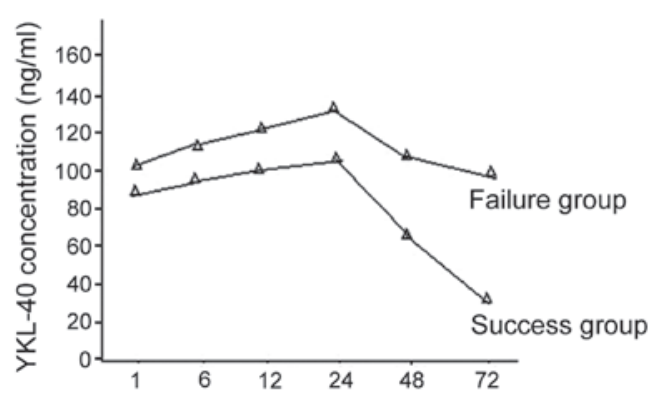

B

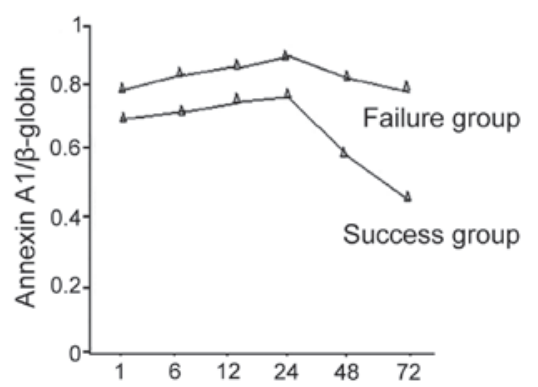

Figure 3. Expression level of YKL-40 and membrane-combined protein A1 at different time points in the success and failure secondary AKI groups. (A) YKL-40 expression level. (B) The relative expression levels of membrane combined protein A1. AKI, acute kidney injury.
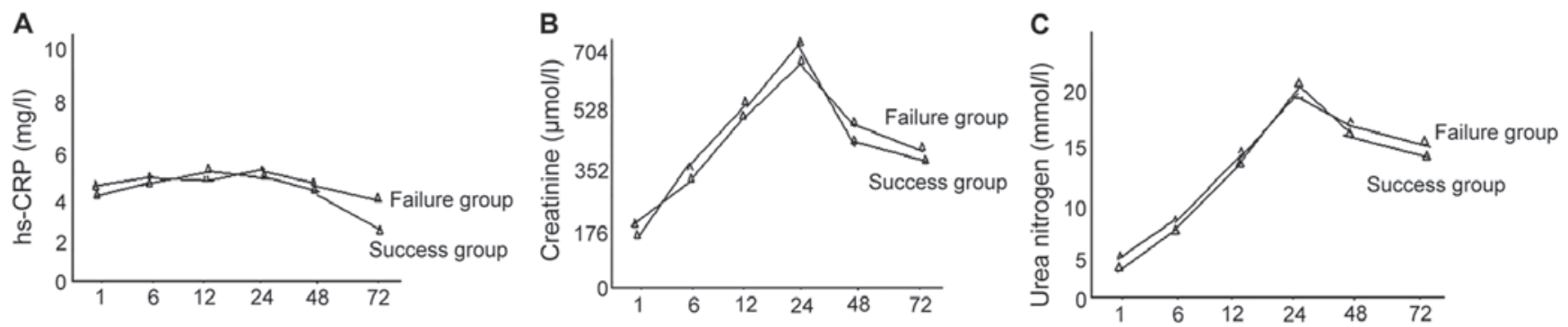

Figure 4. Levels of hs-CRP, serum creatinine and blood urea nitrogen at each time point in the success and failure groups: Level of (A) hs CRP, (B) serum creatinine, and $(\mathrm{C})$ urea nitrogen. 


\section{Discussion}

YKL-40 is a carbohydrate-binding protein belonging to the chitinase family, and has a molecular weight of $40 \mathrm{kDa}$. YKL-40 is present in very low quantities in normal human mononuclear cells (18.9 ng/ml) but may increase significantly after infection (in pyemic non-secondary AKI it reached $62.4 \mathrm{ng} / \mathrm{ml}$ and in the secondary AKI group it reached $105.7 \mathrm{ng} / \mathrm{ml}$ ). Prior studies have shown that for child patients suffering from pyemia, the YKL-40 concentration was reduced by $40 \%$ after $12 \mathrm{~h}$ of CRRT and returned to normal at $24 \mathrm{~h}$ (11). The results of the current study have shown that YKL-40 concentration in pyemic secondary AKI patients increased with time and reached its peak at $24 \mathrm{~h}$. YKL-40 concentration in the failure group was higher than that of the success group at all time-points. The YKL-40 concentration was significantly decreased by CRRT and in some cases the YKL-40 concentration decreased more significantly in the success group. Thus, changes in the YKL-40 concentration may be used as a sensitive index for using CRRT and to predict its treatment effects. YKL-40 secretion may be closely associated with a group of inflammatory cytokines. Findings have shown that excessive release of interleukin (IL)-6 during pyemia was the key factor in enhancing the YKL-40 expression level (12). Activation of the toll-like receptor/NF- $\kappa \mathrm{B}$ signaling pathway and excessive release of pro-inflammatory factors such as IL-1, Il-6 and TNF- $\alpha$ is an important mechanism for YKL-40 secretion.

Annexin A1 is a structure-dependent, calcium-dependent phospholipid-binding protein superfamily. Annexin A1 is a multifunctional protein, involved in the regulation of inflammation, phagocytosis, signal transmission, cell differentiation and apoptosis (13). Annexin A1 is an important regulatory molecule for glucocorticoid in the anti-inflammatory process by: i) preventing the formation of arachidonic acid and inflammatory precursor formation; ii) inducing the production of anti-inflammatory factors by inhibiting phospholipase A2 activity; iii) inhibiting the formation of cyclooxygenase and nitric oxide synthase; iv) activation and emigration of neutrophile granulocytes; and v) promoting the removal of apoptotic cells to avoid necrosis that triggers an inflammatory response (14). The anti-inflammatory action of Annexin A1 is mainly regulated through the FPRs signaling pathway (15).

The Annexin A1 expression level in normal human body is normally low, but is increased in pyemia significantly and is higher in secondary AKI. Annexin A1 is associated with inflammatory response and immune regulation. We observed that the Annexin A1 expression level in pyemic secondary AKI patients increased with time without any obvious peak value. In the failure group, the values of the time-points were significantly higher than those in the success group. Annexin A1 expression was greatly reduced by CRRT and there were more cases of reduced expression in the success group than in the failure group. Thus, changes of Annexin A1 expression are an important index to predict treatment effects, although it was not as sensitive as YKL-40 in terms of guiding the start of CRRT.

There were also no obvious changes in hs-CRP in all the time-points in the pyemic secondary AKI success and failure groups. Creatinine and urea nitrogen levels increased with time but differences in all the time-points were not statistically significant. Following treatment, the hs-CRP level in the success group was significantly reduced while creatinine and urea nitrogen level in the two groups decreased, albeit differences were not statistically significant. Thus, hs-CRP may be used as an important index to predict CRRT effects. It was not ideal to use the creatinine and urea nitrogen level to start CRRT and predict clinical effects. Notably, changes in serum cartilage glycoprotein 39 constitute a sensitive index for using CRRT, which may predict treatment effects together with Annexin A1.

\section{Acknowledgements}

The present study was supported by the National Natural Science Foundation of China (no. 31571187).

\section{References}

1. Zhang P, Yang Y, Lv R, Zhang Y, Xie W and Chen J: Effect of the intensity of continuous renal replacement therapy in patients with sepsis and acute kidney injury: a single-center randomized clinical trial. Nephrol Dial Transplant 27: 967-973, 2012.

2. Wang N, Jiang L, Zhu B, Wen Y and Xi X-M; Beijing Acute Kidney Injury Trial (BAKIT) Workgroup: Fluid balance and mortality in critically ill patients with acute kidney injury: a multicenter prospective epidemiological study. Crit Care 19: 371, 2015.

3. Smith OM, Wald R, Adhikari NKJ, Pope K, Weir MA and Bagshaw SM; Canadian Critical Care Trials Group: Standard versus accelerated initiation of renal replacement therapy in acute kidney injury (STARRT-AKI): study protocol for a randomized controlled trial. Trials 14: 320, 2013.

4. Chou YH, Huang TM, Wu VC, Wang CY, Shiao CC, Lai CF, Tsai HB, Chao CT, Young GH, Wang WJ, et al; NSARF Study Group: Impact of timing of renal replacement therapy initiation on outcome of septic acute kidney injury. Crit Care 15: R134, 2011.

5. Vesconi S, Cruz DN, Fumagalli R, Kindgen-Milles D, Monti G, Marinho A, Mariano F, Formica M, Marchesi M, René R, et al; DOse REsponse Multicentre International collaborative Initiative (DO-RE-MI Study Group): Delivered dose of renal replacement therapy and mortality in critically ill patients with acute kidney injury. Crit Care 13: R57, 2009.

6. Yasuda H, Uchino S, Uji M, Ohnuma T, Namba Y, Katayama S, Kawarazaki H, Toki N, Takeda K, Izawa J, et al; Japanese Society for Physicians and Trainees in Intensive Care Clinical Trial Group: The lower limit of intensity to control uremia during continuous renal replacement therapy. Crit Care 18: 539, 2014.

7. Limaye AP, Kirby KA, Rubenfeld GD, Leisenring WM, Bulger EM, Neff MJ, Gibran NS, Huang ML, Santo Hayes TK, Corey L, et al: Cytomegalovirus reactivation in critically ill immunocompetent patients. JAMA 300: 413-422, 2008.

8. Ronco C, Tetta C, Mariano F, Wratten ML, Bonello M, Bordoni V, Cardona $\mathrm{X}$, Inguaggiato $\mathrm{P}$, Pilotto L, d'Intini V, et al: Interpreting the mechanisms of continuous renal replacement therapy in sepsis: the peak concentration hypothesis. Artif Organs 27: 792-801, 2003.

9. Hattori N, Oda S, Sadahiro T, Nakamura M, Abe R, Shinozaki K, Nomura F, Tomonaga T, Matsushita K, Kodera Y, et al: YKL-40 identified by proteomic analysis as a biomarker of sepsis. Shock 32: 393-400, 2009.

10. Kliger AS, Foley RN, Goldfarb DS, Goldstein SL, Johansen K, Singh A and Szczech L: KDOQI US commentary on the 2012 KDIGO Clinical Practice Guideline for Anemia in CKD. Am J Kidney Dis 62: 849-859, 2013.

11. Schmidt IM, Hall IE, Kale S, Lee S, He CH, Lee Y, Chupp GL, Moeckel GW, Lee CG, Elias JA, et al: Chitinase-like protein Brp-39/YKL-40 modulates the renal response to ischemic injury and predicts delayed allograft function. J Am Soc Nephrol 24: 309-319, 2013. 
12. Zhang L, He H, Wang J and Sheng D: Expression of cartilage glycoprotein 39 in peripheral blood monocytes of septic rat and its role in TLR 4-NF- $\kappa$ B signaling pathways. Int J Clin Exp Med 8: 2459-2464, 2015.

13. Horlacher T, Noti C, de Paz JL, Bindschädler P, Hecht ML, Smith DF, Fukuda MN and Seeberger PH: Characterization of annexin A1 glycan binding reveals binding to highly sulfated glycans with preference for highly sulfated heparan sulfate and heparin. Biochemistry 50: 2650-2659, 2011.
14. McArthur S, Cristante E, Paterno M, Christian H, Roncaroli F, Gillies GE and Solito E: Annexin A1: A central player in the anti-inflammatory and neuroprotective role of microglia. J Immunol 185: 6317-6328, 2010.

15. Brooks AC, Rickards KJ and Cunningham FM: Modulation of equine neutrophil adherence and migration by the annexin-1 derived N-terminal peptide, Ac2-26. Vet Immunol Immunopathol 145: 214-222, 2012. 